\title{
Breaking Mariano Artigas' Frontiers of Evolutionism
}

\section{JAVIER NOVO VILLAVERDE}

Universidad de Navarra

fnovo@unav.es

\begin{abstract}
I review Mariano Artigas' appraisal of Evolution as reflected in some of his works. I find that his perception of evolutionary theory changed from a critical attitude, mainly directed against the modern synthesis of the twentieth century, to a more lenient approach as he incorporated new elements into his reflections. However, he did not fully appreciate some of the advances made in evolutionary biology in recent years. I provide some examples, taken from the field of evolutionary genomics, which shed new light on why evolvability is a necessary property of living beings and how adaptation proceeds through the rewiring of modular gene regulatory networks, resulting in a remarkable degree of phenotypic plasticity. This view provides a richer understanding of two key elements of Artigas' portrayal of the modern worldview: dynamism and patterning.
\end{abstract}

Keywords: Mariano Artigas; Evolution; genomics; philosophy of biology.

\section{Introduction}

The work of Mariano Artigas was an effort to bridge the increasingly wider gap between the explanation of the cosmos offered by modern science and the image of the universe that is portrayed by religion, as a divine plan that unfolds through natural processes. One of the main themes in his writings is that science will never have the last (or exclusive) word in this dispute, because it cannot explain the very conditions of possibility on which it rests.

Being a physicist turned into philosopher of science, Artigas' depiction of the worldview that science has built over the last century mainly deals 
with issues arising from physics. When he refers to nature in many passages of his works, it seems that he is thinking mostly about inanimate beings. However, since biological evolution is often used as an argument in favour of naturalism, some of his early works deal with this scientific theory. His first book on evolution was published in 1985 under the title Las fronteras del evolucionismo (the frontiers of evolutionism). The fifth edition, published in 1991, included an addendum in which he commented on some recent scientific advances. This work was published in a revised form in 2004, with several changes to chapter titles and some new chapters introduced. As it turned out, this was the last edition of the book before his death in 2006. In some of his other works he also touched upon evolution, notably in The Mind of the Universe and in Filosofía de la Naturaleza (philosophy of nature). In these two books he repeats more or less the same philosophical arguments about the explanatory power of evolutionary theory. In a historical context where evolution was portrayed as the ultimate proof against the existence of a divine plan for the universe, his main line of argumentation was to show that such claim falls beyond the limits of experimental science.

It is interesting to note that, for Artigas, the "frontiers of evolutionism" are in fact issues related to philosophy of science, such as whether science will ever be able to explain divine action in the world, the creation of the universe ex nihilo or the human soul, as well as issues such as finality and chance. It is clear that these topics have very little to do with standard evolutionary theory, so the original title of the book is a bit of a misnomer as he himself acknowledges in the addendum to the fifth edition of 1991. In the same vein, it is interesting to note the use of the word "Evolutionism" in all his works in Spanish. He will only use the less ideology-charged word "Evolution" in the English edition of The Mind of the Universe $(2001,115)$.

\section{The uncomfortable relationship of Artigas with the Modern Synthesis}

I believe that Artigas' problems with evolutionary theory can be better understood in the light of the previous considerations. He set out to show 
that evolution, like any other scientific theory, could not claim to be the only and ultimate explanation of the natural world. As many philosophers of science, the path he chose to do this was to underscore the gaps in standard evolutionary theory. The problem with this approach is that (like many philosophers of science) he was not an expert evolutionary biologist, so many of his criticisms either lack enough depth or refer to problems that have been settled in the field. A case in point is his attack on what is known as the "modern evolutionary synthesis".

Given that his first book on evolution dates back to 1985, the evolutionary theory that Artigas chastises in his writings is the neo-darwinian synthesis developed in the middle of the $20^{\text {th }}$ century, which relies on the mutation/selection paradigm as the most powerful (if not the only) evolutionary force. Other basic tenets of this modern synthesis (as it was called at the time) are gradualism, the belief that morphological evolution in anatomy is the result of small incremental changes over time, and adaptationism, the tendency to explain all phenotypic traits as the result of adaptation to environmental conditions. However, after Kimura's neutral theory of molecular evolution, the molecular clock hypothesis led to the recognition that neutrality, not selection, is the best null hypothesis in molecular evolution studies, thus dealing a death blow to panselectionism (Dietrich 2013). Adaptationism was attacked by Gould and Vrba in 1982, with their proposal that many morphological innovations could be explained by the co-option of pre-existing traits or functions (exaptations) in order to solve biological problems, instead of relying on naive adaptationist explanations. More recently, in the field of comparative genomics Koonin has persuasively shown the insufficiency of the gradualist neo-darwinian paradigm to explain evolutionary changes in form and function and the appearance of new body plans (Koonin 2008). Today it is generally accepted that instead of a gradual trend towards increasing genome complexity, genome expansion and complexification has been the result of certain episodes of genomic duplication, sub-functionalization and co-option of pre-existing genetic modules, during which neutral or nearly-neutral innovations became fixed thanks to the relaxing of purifying selection in populations of small effective 
size. In other words, episodes of rapid increase in genome complexity were mostly non-adaptive (Lynch 2007).

Despite of all these new insights that have extended the classical neo-darwinian evolutionary synthesis, Artigas' efforts were mainly directed to show the inconsistencies or insufficiencies of the neo-darwinian selectionist paradigm that prevailed until the 1980s. In The Mind of the Universe, published as late as 2000, Artigas criticizes neo-darwinian thinking without any reference to the neutral theory of molecular evolution or to the field of developmental evolutionary biology ("evo-devo"). In his 2003 book on philosophy of nature he already mentions Kimura's neutral theory and other modern concepts such as gene regulatory networks (surprisingly, throwing them together with Stuart Kauffman's theories of self-organization and selection in evolution), but there is still no mention of the relevance of developmental biology, evolutionary convergence, comparative genomics or ecological constraints.

One would have expected to find these ideas discussed in his last book on evolution, the 2004 edition of Las fronteras del evolucionismo, in which Artigas includes a whole new chapter on "La evolución del evolucionismo". However, it is somehow disappointing that he focuses on a critique of Mayr's 2001 edition of What Evolution Is and Gould's The Structure of Evolutionary Theory, published in 2002. In my opinion, this shows that he was more comfortable discussing classical darwinian theory than incorporating recent advances in evolutionary biology to his argument. For instance, Artigas is very critical of adaptation as a universal explanation for every trait, so I find it hard to understand that he fails to comment on "The Spandrels of San Marco", the hotly debated 1979 paper by Gould and Lewontin in which they deal precisely with this issue and criticize the adaptationist narrative in evolutionary literature.

In fact, there is at present a hot debate in the field of evolutionary biology as to whether the evolutionary synthesis needs a rethink or not (Laland et al. 2014). It might be argued that this discussion unfolded after the death of Artigas in 2006, but the ideas that are now being debated had been circulating in the scientific literature for some years. As I said before, 
I believe that he was not aware of the full extent of the "evolution of evolutionary theory" over the last decades and that he missed the subtleties of these debates because he was not as conversant with evolutionary biology as he was with topics related to physics.

Judging from his writings it is fair to say that, over the time, Artigas took a more lenient approach towards evolutionary explanations. Despite his personal evolution on this matter, however, he never reached a full understanding of modern evolutionary theory as it is formulated in the $21^{\text {st }}$ century. Here I will try to explain how recent developments in evolutionary biology, particularly in the field of comparative genomics, fill some of the gaps and insufficiencies that Artigas exposed in his critique of the neo-darwinian evolutionary synthesis.

\section{Random mutations and selection}

One of the main points of neo-darwinian theory that is attacked by theist philosophers of science is the "randomness" of mutations and the "blind" behaviour of natural selection, and Artigas was no exception to this. However, in The Mind of the Universe he makes an interesting observation about genetic variation and natural selection as a cause of order in nature:

Natural selection should be considered a cumulative process and not purely random play. Indeed, when a certain level of organization has been reached, genetic variations and the subsequent natural selection act within the restricted range of possibilities permitted by that present organization. As natural selection progresses, many possibilities will be discarded and new possibilities will arise. (Artigas 2001, 116)

He concludes that the probability of arriving at a highly organized state increases when previous steps have already led to organized entities and processes. To him this shows the existence of some intrinsic principles of organization so that not everything can be explained by blind forces. A similar concept in evolutionary biology is that of "constraint". 
In another passage of The Mind of the Universe Artigas is discussing the role of chance and lawfulness to create patterns and self-organization. He proposes that many cooperative features of nature have yet to be discovered, particularly in the field of evolutionary theory:

Despite the claims of orthodox Darwinians who consider natural selection as the chief cause of evolution, other scientists continue searching for new structural laws that may help bridge the many gaps within the evolutionary explanations. Empirical research has found some promising clues in the field of genetics, where the complex combinations of different levels of genetic regulation could explain how a particular mutation can sometimes produce big and yet viable change. (Artigas 2001, 95)

This is a particularly interesting insight, and I find it surprising that Artigas did not develop this idea further in later works, particularly in the last edition of Las fronteras del evolucionismo. Had he been more familiar with advances in evolutionary genomics, he would have found that some of those "structural laws" are well known to evolutionary theory. In fact, we know that not all genetic mutations are equally relevant to natural selection. To understand this, one must consider that the levels of generality and specificity found in nature are the result of the evolutionary history of organisms. As organisms diversify they will share many properties, more at the beginning, less as their lineages progress along their own separate ways. Descent (historical relatedness) and definition (shared properties) are two closely intertwined aspects of the same process of causation. For the biologist who considers the evolutionary past, the particular morphology and physiology of an organism are precisely the result of its evolutionary trajectory. The "intrinsic" properties of an organism are inseparable from its particular evolutionary history. Therefore, considering the evolutionary past is the best way to understand what an organism is and why it has developed a particular set of properties. The question "why does a specific cluster of properties coexist in a given organism?" can only be answered in the light of the evolutionary past of that organism. 
Two properties of mutations are very important in this regard, because they can explain not only historical constraints but also how some mutations can "produce big and yet viable change". These properties are epistasis and pleiotropy. Epistasis is a type of genetic interaction by which the combined phenotypic effect of two or more genes is weaker (negative epistasis) or stronger (positive epistasis) than the additive effects of each gene. Therefore, it is like the genetic "memory" of the evolutionary history of organisms. Pleiotropy refers to mutations with multiple phenotypic effects ("big” mutations in Artigas' words). As expected, they are rarely viable (particularly when they affect developmental genes), so they should be eliminated by natural selection and hardly contribute to evolutionary adaptation. I will address first how epistasis can favour certain mutations during evolutionary change, whereas the role of pleiotropy will be present in several discussions throughout the rest of this article. I am sure that Artigas would have found these concepts very useful.

\section{Macroevolution in the light of genomics}

In biology, form is generally understood as body plan (bauplan), that is, the external shape, internal anatomy and particular physiology of an organism (classically referred to as "form and function"). These features are the result of a very specific developmental program encoded in the genome and deployed through transcriptional and regulatory networks that interact with environmental cues in a particular ecological context. As mentioned above, the gradualist neo-darwinian paradigm cannot satisfactorily account for such changes, so explaining how body plans evolve is one of the major challenges of evolutionary biology.

The need to understand macroevolutionary transitions and the discovery of "macromutations", such as mutations in homeotic genes ${ }^{1}$, led Goldschmidt (1940) to propose the concept of "hopeful monsters" to explain macroevolution. Even though experimentally induced macromutations

1 Homeotic genes encode proteins involved in the formation of basic body structures during development. 
resemble evolutionary transitions, no such mutations have been shown to account for the appearance of body plans, as they would involve large pleiotropic and epistatic effects. It was later demonstrated that mutations with pleiotropic and epistatic effects, especially if they affect developmental genes, usually decrease fitness and are eliminated by natural selection, so they will very rarely become fixed in large populations. Therefore, the concepts "macromutation" and "hopeful monsters" were abandoned. In the light of genomic studies performed over the last decade, we are now closer to a global explanation of the evolution of form that includes the relative contribution of gene duplication and non-coding regulatory DNA, given the constraints imposed by pleiotropy and epistasis.

\subsection{Epistasis and evolutionary history}

Mutagenesis experiments have shown that, while many different mutations can lead to similar phenotypic changes in the laboratory, only a subset of them are found in naturally evolving populations (Stern 2011, 4). One of the reasons for this is the presence of epistatic effects. In the context of evolution, epistasis explains the historical memory of organisms, because a mutation can display distinct phenotypic effects depending on its particular evolutionary trajectory (that is, the number of potential epistatic mutations that the organism has acquired throughout its evolutionary history). Previous mutations affecting developmental pathways could mask (or unmask) phenotypic effects of new mutations. Using a graphical example, mutations in a gene called ultrabithorax lead to the appearance of a new thoracic segment with an additional pair of wings in flies, but cannot have the same result in a primate due to the vastly different developmental programs that primates and insects have configured throughout their separate evolutionary trajectories. In other words, the evolutionary past imposes clear constraints on what an organism can and cannot become.

A recent example that helps to illustrate the importance of epistasis in evolution is the dissection of the molecular changes that led to trichromatic vision in primates over the last 90 million years (My). The blue-sensitive 
visual pigment in humans evolved from the UV-sensitive pigment in the Boreoeutherian ancestor by seven mutations. During the period between 45 and 30 My ago, when the human pigment was in the final stage of developing its blue-sensitivity, two red-sensitive pigments appeared by gene duplication and one of them became green-sensitive. Thus, trichromatic colour vision in the human lineage was fully developed by $30 \mathrm{My}$ ago through inter-protein epistasis among the three visual pigments, in such a way that the evolutionary pathway could only be completed when several of the changes combined in a particular sequence of events (Yokoyama et al. 2014).

\subsection{Relaxing pleiotropy by gene duplication}

The comparison of sequenced genomes from many different species has shown the importance of gene duplication as a major evolutionary mechanism. After a gene is duplicated (an event that cannot be considered "gradual"), one of the copies of the gene usually retains its original function whereas the other copy can be neo-functionalized (that is, mutated so that it serves a novel function) or sub-functionalized (so that its expression is restricted to one or a few tissues). The presence of an intact original copy of the gene relaxes the strength of purifying selection on the other copy so that even pleiotropic mutations in developmental genes could bypass pleiotropy and become fixed, especially in populations of small size. A very good example is the burst of segmental duplications that took place in the genomes of African Great Apes which is thought to have played an important role in extending the repertoire of gene functions required for the evolution of the human brain (Marques-Bonet et al. 2009). Recently, one of these duplications (involving a gene known as $S R G A P 2$ ) was studied in greater detail (Dennis et al. 2012). As a result of three consecutive duplications, there are four different copies of $S R G A P 2$ in the human genome. The original copy (known as $S R G A P 2 A$ ) underwent a duplication 3.4 My ago, an event that created a copy called $S R G A P 2 B$. Later, SRGAP2B duplicated twice independently to originate SRGAP2C (2.4 My ago) and SRGAP2D (1 My ago). What is interesting is that the protein encoded by SRGAP2A 
prevents synapse formation in the cortex of the brain, whereas SRGAP2C has the opposite effect. In fact, when scientists introduced SRGAP2C in mice (which only have the original copy SRGAP2A, but do not have SRGAP2C) synapse formation accelerated in the brains of the animals. The temporal coincidence of this duplication with the first recognizable stone tools made by humans (about $2.5 \mathrm{My}$ ago) and with the increase in brain size that we see in hominin fossils suggests that it may also have contributed to shape the human brain.

Another example of the crucial role of duplications in the evolution of form comes from the sequencing of the genome of the elephant shark (Callorhinchus milii), which revealed that the replacement of cartilage in cartilaginous fish (chondrichthyes) for bone in fish with bony skeletons (osteichthyes) could have been the result of a series of duplications involving a gene called Sparc in the ancestor of sharks and bony fish (Venkatesh et al. 2014).The initial duplication of Sparc gave rise to another gene called Sparcl1. Later, a series of tandem duplications of Sparcl1 in the bony fish ancestor created a new gene family known as SCPP, which is responsible for bone formation. When scientists knocked down some of the genes belonging to this gene family in zebrafish embryos, their skeletons lost bone, supporting a role in bone formation in osteichthyes.

\subsection{Non-coding regulatory elements}

However, mutations that affect the protein-coding region of genes seem to be rare in developmental genes, particularly during the evolution of body plans. To explain this, King and Wilson proposed in 1975 that evolutionary changes in anatomy would be based preferentially on changes in the control of gene expression (King and Wilson 1975). Thirty years later, after reviewing the literature, Carroll argued that there was an "adequate basis to conclude that the evolution of anatomy occurs primarily through changes in regulatory sequences" (Carroll 2005). Genomic analyses performed over the last decade have only reinforced the view that evolution of non-coding regulatory DNA is a major contributor to the evolution of form. 
Large-scale genomic projects undertaken over the last few years have identified thousands of regulatory regions in the human genome (Bernstein et al.2012). Such regions behave as enhancers or silencers of gene expression and can be defined by specific epigenetic modifications, chromatin accessibility and occupancy by transcription factors. Notably, a large proportion of them are highly conserved in the genomes of many animal groups. These Conserved Non-coding Elements (CNEs) represent a set of transcriptional enhancers that regulate the expression of genes coding for proteins involved in developmental pathways (Vavouri and Lehner 2009).

Conserved non-coding regulatory sequences have all the characteristics that make them ideal candidates to explain the diversification of animal body plans. First, they regulate the expression of developmental genes, which greatly expanded during the period of rapid diversification of animal body plans (Larroux et al. 2008). This expansion in the repertoire of developmental genes led to a high degree of complexification of developmental networks. However, the same basic set of regulatory genes controls development in different animal groups. Therefore, different animal morphologies must be explained by the re-deployment of the same genes in various alternative regulatory circuits, leading to gene-regulatory networks of different topologies (Erwin and Davidson 2009). Extensive rewiring of these gene-regulatory networks during the early period of animal evolution led to the appearance of the core developmental programs of extant major animal phyla. Since then, body plans in animals have been highly conserved, with the result that CNEs are evolving very slowly and are highly conserved within each animal group (Vavouri et al. 2007). Consistent with this, a comprehensive search for non-coding regulatory elements conserved between protostomes and deuterostomes found only two examples, suggesting that the deployment of novel developmental programs necessary for deuterostome development would have been mostly the result of rewiring pre-existing genetic networks (Clarke et al. 2012).

An impressive example of the fact that changes in conserved non-coding regions of the genome mirror macroevolutionary trends in morphology and anatomy comes from a recent study by Lowe et al. Comparing forty 
vertebrate genomes, they identified several million CNEs. They could also determine when such elements became regulatory over the last $600 \mathrm{My}$ and which genes they regulate (Lowe et al. 2011). Notably, the most ancient set of CNEs (those that became active between 600 and 300 My ago) regulate the activity of transcription factor and developmental genes, confirming the extensive rewiring of developmental networks that took place at the time of diversification of animal body plans.

More recent studies are only adding weight to the hypothesis that evolutionary innovations in form and function have been primarily the result of changes in regulatory sequences. The transition from water to land by tetrapods during the Devonian required the loss of the fin rays and the emergence of an autopod with digits in the tetrapod limb. The insertion of a regulatory element from the mouse HOXD cluster in zebrafish embryos increased hoxd13 activity and recapitulated both of these changes (Freitas et al. 2012).

Similar findings have been made with regard to the evolution of the human brain. Reilly et al. (2015) have recently identified thousands of regulatory elements involved in the formation of the neocortex during the first weeks of human embryonic development, which however do not display such activity in rhesus macaque brains. Another remarkable example is the finding that a human CNE regulating the expression of a neurodevelopmental gene, when inserted in the mouse genome, leads to a $12 \%$ increase in brain size in mice, whereas the chimpanzee version of such element does not (Boyd et al. 2015). These recent studies support the notion that macro-evolutionary transitions in form are the result of changes in non-coding elements that control the expression of developmental genes.

\section{Tinkering your way to evolutionary success}

Throughout his writings, Artigas was adamant that the present worldview shows a grandiose process of self-organization driven by information, in which an internal dynamism is constantly creating new spatial and temporal patterns. Thus, he pays special attention to patterns, of which he says that 
are "closely tied to the ancient concept of form as it was used to refer to the modes of being of the different entities". For Artigas, patterning is a key feature of the present worldview:

The process takes places usually as the production of individual patterns that reproduce a general type that already exists, but in special circumstances it may provoke a new outcome, and a new type of natural pattern is formed and eventually retained and reproduced. Both types of patterning are creative [...]. In both cases the phenomenon is really remarkable, especially when we realize that the construction of our world has required an immense quantity of successive processes of patterning, each one of which has opened the door to new potentialities that have later been realized. (Artigas 2001, 93)

He understands that this is particularly clear in the case of living beings, characterized by a special type of dynamism and patterning that lead to strong internal unity and individuality. When nature is viewed through this perspective of dynamism and patterning, evolution can be considered as a series of morphogenetic processes so that at each step some potentialities are actualized depending on the factors at play. This leads to new modes of organization that contain novel types of dynamism and potentialities, whose deployment results in new levels of organization and so on. This process can be regarded as "the deployment of information which, in successive levels of organization, results in new informational patterns of increasing complexity" (Artigas 2003, 257).

This idea of form is clearly consistent with the view of form that we have depicted in the previous section. In the light of evolutionary genomics, form can be adequately understood in terms of information deployed through developmental programs encoded in modular genetic networks. During evolution, new forms arise when such networks change and adapt to specific environmental cues, within the constraints of historical developmental trajectories. Thus, the evolution of form and function can be described as the process whereby developmental programs are modified through the rewiring of gene regulatory networks, by processes such as gene duplication or by changes in non-coding regulatory sequences. 
In addition, the fact that organisms are constituted by modular gene regulatory networks is the basis of phenotypic plasticity and this, in turn, explains the mutability and stability required for adaptive evolution. In other words, it explains why organisms are capable of evolving and why evolution is adaptive. What this means is that evolvability is a necessary property of all living beings. This is a very important insight, but unfortunately Artigas was not aware of its relevance, or at least did not discuss it at length in his works.

All this is closely linked to another important concept in Artigas' philosophy of nature, which is the relationship between finality, predictability and the role of contingency. Again, it is surprising that he never mentioned some of the great debates that took place on this issue within the field of evolutionary biology, such as Gould's defence of contingency in Wonderful Life (1990) and the reply by Conway-Morris (1998). In The Mind of the Universe, however, there is a whole section on divine action where he tries to show that teleology, contingency and necessity can be made compatible in the light of chaos theory (Artigas 2001, 145), which eloquently shows his bias towards problems and examples taken from the field of physics.

His omission of arguments arising from evolutionary biology is all the more surprising, because the concepts that I have illustrated throughout this article are crucial in any discussion about finality and contingency. On the one hand, the new evolutionary synthesis departs from the gradualist dogma and portrays evolution as a tinkerer, whereby extant elements (genes or regulatory modules) are co-opted and combined in novel ways so that they can provide new solutions to biological problems. But this also means that the number of possible combinations is limited, so that within this scenario full of contingencies similar phenotypic solutions are frequently reached by independent paths, a situation known as convergent evolution. The pervasive role of phenotypic convergence in evolution has been reviewed elsewhere (Conway-Morris 2003; McGhee 2011) and recent genomic research is shedding new light on the mechanisms leading to molecular convergence at the genetic level (Parker et al. 2013). Collectively, such studies have established the role of regulatory network structure in generating hotspots of molecular convergent evolution (Martin and Orgogozo 2013) and 
suggest that genetic evolution can be historically predictable (Stern 2011). For instance, the structure of developmental networks helps to explain why some genes are more likely to underlie phenotypic evolution than others (Stern 2013). Genes which behave as evolutionary hot-spots are usually hubs in gene-regulatory developmental networks. When such networks are viewed across evolutionary time, mutations that maximize fitness with minimal pleiotropic effects are more likely to be recurrent. In other words, the probability that an adaptive mutation is fixed largely depends on how it alters development to generate phenotypic change: this is the main developmental constraint in evolutionary change at the genetic level (Stern 2011, 175 ff).

\section{Conclusion}

Given that Artigas' work is an attempt to show that natural processes, full of contingencies, can be reconciled with divine action and finality, I wonder how he would have incorporated the lessons learned from evolutionary genomics into his argument. He was very fond of a particular passage in Aquinas' Commentary to Aristotle's Physics, (In Physic., lib. 2 I. 14 n. 8), where Aquinas defines nature as "a certain kind of art [...] impressed upon things, by which these things are moved to a determinate end. It is as if the shipbuilder were able to give to timbers that by which they would move themselves to take the form of a ship". This passage reveals a strikingly prescient view of the evolutionary nature of organisms, which can produce new forms by themselves, that is, by their own biological structure. Artigas found it "breath-taking", as it "corresponds literally to the kind of self-organization that today we study scientifically" (Artigas 2001, 156). For him, the new worldview paves the way for a kind of "comprehensive naturalism" in which the role of natural agency is fully recognized, while at the same time supported by a founding divine action. I believe that the latest advances in evolutionary biology would have helped him greatly to progress towards that view. 


\section{References}

Artigas, M. 1991. Las fronteras del evolucionismo. Madrid: Ediciones Palabra. Reedition 2004. Pamplona: Eunsa.

-. 2001. The Mind of the Universe: Understanding Science and Religion. Radnor, Penn:

Templeton Foundation Press.

-. 2003. Filosofía de la naturaleza. Pamplona: Eunsa.

Bernstein, B. E., E. Birney, I. Dunham, E. D. Green, C. Gunter, and M. Snyder. 2012. "An Integrated Encyclopedia of DNA Elements in the Human Genome." Nature 489:57-74. doi:10.1038/nature11247.

Boyd, J. L., S. L. Skove, J. P. Rouanet, L. J. Pilaz, T. Bepler, R. Gordân, G. A. Wray, and D. L. Silver. 2015. "Human-Chimpanzee Differences in a FZD8 Enhancer Alter Cell-Cycle Dynamics in the Developing Neocortex.” Current Biology 25:772-779. doi:10.1016/j.cub.2015.01.041.

Carroll, S. B. 2005. "Evolution at Two Levels: On Genes and Form.” PLoS Biology 3:1159-1166. doi:10.1371/journal.pbio.0030245.

Clarke, S. L., J. E. Vandermeer, A. M. Wenger, B. T. Schaar, N. Ahituv, and Gill Bejerano. 2012. "Human Developmental Enhancers Conserved between Deuterostomes and Protostomes.” PLoS Genetics 8/8:e1002852. doi:10.1371/journal.pgen.1002852.

Conway-Morris, S. 1998. The Crucible of Creation: The Burgess Shale and the Rise of Animals. Oxford: Oxford University Press.

-. 2003. Life's Solution: Inevitable Humans in a Lonely Universe. Cambridge, UK; New York: Cambridge University Press.

Dennis, M. Y., X. Nuttle, P. H. Sudmant, F. Antonacci, T. A. Graves, M. Nefedov, J. A. Rosenfeld, S. Sajjadian, M. Malig, H. Kotkiewicz, C. J. Curry, S. Shafer, L. G. Shaffer, P. J. de Jong, R. K. Wilson, and E. E. Eichler. 2012. "Evolution of Human-Specific Neural SRGAP2 Genes by Incomplete Segmental Duplication.”Cell 149:912-922. doi:10.1016/j.cell.2012.03.033.

Dietrich, M. R. 2013. “Molecular Evolution.” In The Philosophy of Biology: A Companion for Educators, edited by K. Kampourakis, 239-248. Dordrecht: Springer Netherlands. doi:10.1007/978-94-007-6537-5_12.

Erwin, D. H., and E. H. Davidson. 2009. “The Evolution of Hierarchical Gene Regulatory Networks.” Nature Reviews. Genetics 10:141-148. doi:10.1038/nrg2499.

Freitas, R., C. Gómez-Marín, J. M. Wilson, F. Casares, and J. L. Gómez-Skarmeta. 2012. "Hoxd13 Contribution to the Evolution of Vertebrate Appendages." Developmental Cell 23:1219-1229. doi:10.1016/j.devcel.2012.10.015.

Goldschmidt, R. B. 1940. The Material Basis of Evolution. New Haven: Yale University Press. 
Gould, S. J. 1990. Wonderful Life: The Burgess Shale and the Nature of History. New York: W. W. Norton \& Company.

King, M. C., and A. C. Wilson. 1975. “Evolution at Two Levels in Humans and Chimpanzees.” Science 188:107-116.

Koonin, E. V. 2008. "Darwinian Evolution in the Light of Genomics.” Nucleic Acids Research 37:1011-1034. doi:10.1093/nar/gkp089.

Laland, K., T. Uller, M. Feldman, K. Sterelny, G. B. Müller, A. Moczek, E. Jablonka, J. Odling-Smee, G. A. Wray, H. E. Hoekstra, D. J. Futuyma, R. E. Lenski, T. F. C. Mackay, D. Schluter, and J. E. Strassmann. 2014. “Does Evolutionary Theory Need a Rethink?” Nature 514:161-164. doi:10.1038/514161a.

Larroux, C., G. N. Luke, P. Koopman, D. S. Rokhsar, S. M. Shimeld, and B. M. Degnan. 2008. "Genesis and Expansion of Metazoan Transcription Factor Gene Classes.” Molecular Biology and Evolution 25:980-996. doi:10.1093/molbev/msn047.

Lowe, C. B., M. Kellis, A. Siepel, B. J. Raney, M. Clamp, S. R. Salama, D. M. Kingsley, K. Lindblad-Toh, and D. Haussler. 2011. “Three Periods of Regulatory Innovation during Vertebrate Evolution.” Science 333:1019-1024. doi:10.1126/ science.1202702.

Lynch, M. 2007. The Origins of Genome Architecture. Sunderland, Mass.: Sinauer Associates.

Marques-Bonet, T., J. M. Kidd, M. Ventura, T. A. Graves, Z. Cheng, L. W. Hillier, Z. Jiang, C. Baker, R. Malfavon-Borja, L. A. Fulton, C. Alkan, G. Aksay, S. Girirajan, P. Siswara, L. Chen, M. F. Cardone, A. Navarro, E. R. Mardis, R. K. Wilson and E. E. Eichler. 2009. "A Burst of Segmental Duplications in the Genome of the African Great Ape Ancestor.” Nature 457:877-881. doi:10.1038/nature07744.

Martin, A., and V. Orgogozo. 2013. “The Loci of Repeated Evolution: A Catalog of Genetic Hotspots of Phenotypic Variation.” Evolution 67:1235-1250. doi:10.1111/ evo.12081.

McGhee, G. R. 2011. Convergent Evolution: Limited Forms Most Beautiful. Cambridge, Mass.: MIT Press.

Parker, J., G. Tsagkogeorga, J. A. Cotton, Y. Liu, P. Provero, E. Stupka, and S. J. Rossiter. 2013. “Genome-Wide Signatures of Convergent Evolution in Echolocating Mammals.” Nature 502:1-9. doi:10.1038/nature12511.

Reilly, S. K., J. Yin, A. E. Ayoub, D. Emera, J. Leng, J. Cotney, R. Sarro, P. Rakic, and J. P. Noonan. 2015. "Evolutionary Changes in Promoter and Enhancer Activity during Human Corticogenesis.” Science 347:1155-1159. doi:10.1126/ science.1260943.

Stern, D. L. 2011. Evolution, Development and the Predictable Genome. Roberts and Company. 
-. 2013. "The Genetic Causes of Convergent Evolution.” Nature Reviews. Genetics 14:751-764. doi:10.1038/nrg3483.

Vavouri, T., and B. Lehner. 2009. "Conserved Noncoding Elements and the Evolution of Animal Body Plans.” BioEssays 31:727-735. doi:10.1002/bies.200900014.

Vavouri, T., K. Walter, W. R. Gilks, B. Lehner, and G. Elgar. 2007. "Parallel Evolution of Conserved Non-Coding Elements That Target a Common Set of Developmental Regulatory Genes from Worms to Humans." Genome Biology 8: 1-14. doi:10.1186/ gb-2007-8-2-r15.

Venkatesh, B., A. P. Lee, V. Ravi, A. K. Maurya, M. M. Lian, J. B. Swann, Y. Ohta, M. F. Flajnik, Y. Sutoh, M. Kasahara, S. Hoon, V. Gangu, S. W. Roy, M. Irimia, V. Korzh, I. Kondrychyn, Z. W. Lim, B. H. Tay, S. Tohari, K. W. Kong, S. Ho, B. Lorente-Galdos, J. Quilez, T. Marques-Bonet, B. J. Raney, P. W. Ingham, A. Tay, L. W. Hillier, P. Minx, T. Boehm, R. K. Wilson, S. Brenner and W. C. Warren. 2014. "Elephant Shark Genome Provides Unique Insights into Gnathostome Evolution." Nature 505: 174-179. doi:10.1038/nature12826.

Yokoyama, S., J. Xing, Y. Liu, D. Faggionato, A. Altun, and W. T. Starmer. 2014. "Epistatic Adaptive Evolution of Human Color Vision.” PLoS Genetics 10/12:e1004884. doi:10.1371/journal.pgen.1004884. 